Denniston, James C., Robert P. Cole, and Ralph R. Miller. (1998). "The role of temporal relationships in the transfer of conditioned inhibition." Journal of Experimental Psychology: Animal Behavior Processes 24, no. 2: 200-214. American Psychological Association (ISSN: 1939-2184) DOI: 10.1037/0097-7403.24.2.200. April 1998.

Keywords: Conditioned Stimulus | Conditioned Suppression | Time | Transfer (Learning) | Rats

\title{
The Role of Temporal Relationships in the Transfer of Conditioned Inhibition
}

\author{
James C. Denniston, Robert P. Cole, \& Ralph R. Miller
}

\begin{abstract}
Two experiments with rats investigated the temporal relationships under which conditioned inhibition will transfer to an independently conditioned excitor (CS) in a summation test. Experiment 1 trained 2 simultaneous inhibitors with either a trace or delay excitatory CS. Transfer of inhibitory behavioral control depended on the temporal relationship of the transfer CS to the unconditioned stimulus (US). Experiment 2 extended these findings by training 2 inhibitors ( 1 simultaneous and 1 serial) with a single delay excitatory CS. Again, testing with trace and delay transfer CSs found that transfer of inhibitor control depended on the temporal relationship of the transfer CS to the US. In both studies, maximal inhibition was observed when the inhibitor signaled US omission at the same time as the transfer excitor signaled US presentation. The results are discussed in terms of the temporal coding hypothesis.
\end{abstract}

\footnotetext{
ARTICLE

Conditioned inhibition is a behavioral phenomenon first reported by Pavlov (1927). In Pavlov's procedure, a conditioned stimulus (CS) is paired with an unconditioned stimulus (US) on some trials (i.e., A+, with A denoting the CS and + denoting the US), while on other trials, a simultaneous compound stimulus consisting of $\mathrm{A}$ and another stimulus $(\mathrm{X})$ is followed by the absence of the US (i.e., AX-, with - denoting nonreinforcement). Following this training, the inhibitory properties of $X$ can be assessed through a summation test in which $X$ is paired with an independently trained CS (B) and conditioned inhibition is evidenced in reduced behavioral control by the compound (i.e., BX) relative to the transfer excitor alone (e.g., B). Recently, students of animal learning and behavior have become interested in the associative structures underlying Pavlovian conditioned inhibition and the factors that mediate the transfer of inhibitory behavioral control to other CSs.
} 
A central question is whether the informational content of a Pavlovian conditioned inhibitor includes the identity of the US, or merely some degraded aspect of the information provided in training such as the affect evoked by the US. If inhibitors encode specific information about the US that was paired with their training excitors, one factor that might determine the degree to which a conditioned inhibitor will transfer its behavioral control to another stimulus is the similarity of the representation of the (omitted) US encoded by the inhibitor (based on its training excitor) to the representation of the anticipated US encoded by the transfer CS. Animals appear to encode several attributes of a US. For example, Colwill and Rescorla (1988) found that a discriminative stimulus can enhance responding to a new instrumental response, provided that the new response is reinforced with the same reinforcer with which the $S^{\mathrm{D}}$ was established. Similarly, Colwill (1991) found that a negative discriminative stimulus can suppress responding on a different response manipulandum, if the new response is trained with the same reinforcer (Experiment 1), and retard acquisition of a new response reinforced with the same reinforcer but not with a different reinforcer (Experiment 2). Collectively, these studies suggest that associations in instrumental situations encode the identity of the specific US that was used in training.

Studies of excitatory Pavlovian conditioning have revealed similar effects to those observed by Colwill (1991) and Colwill and Rescorla (1988) in instrumental preparations. For example, Colwill and Motzkin (1994, Experiment 2) found that presentation of a Pavlovian CS that signals a forthcoming US has a debilitating effect on the rate of an instrumental response that was reinforced with a different outcome. These results support the notion that Pavlovian CSs, in addition to instrumental discriminative stimuli, provide qualitative information about the identity of the reinforcer.

The work of Pavlov (1927) suggests that time might be an encoded attribute of a CS-US association. Pavlov's work on inhibition of delay revealed that when CSs of long duration were used as signals for the US in salivary conditioning, the conditioned response was restricted to the later portion of the CS (i.e., just prior to US onset). Moreover, Rescorla (1967) extended the findings of Pavlov to fear conditioning using a Sidman avoidance task. These findings lend support to the notion that onset of a CS provides information about the temporal location of the forthcoming US which may then guide the initiation of the conditioned response.

The concept of temporal coding of US presentations has been explored by several other researchers in recent years. For example, Goddard (1995) measured the rate of magazine entry by rats that received food USs on a fixed-time schedule of reinforcement, and observed reduced magazine entry following the US presentation (Experiment 1), and an increased rate of magazine entry near the time at which the next US was expected (Experiment 2). Roberts (1981), using the peak procedure, found that maximal response rates occur at the time at which US presentation was expected following fixed-time schedules of reinforcement. Furthermore, Holland (1980) observed that the topology of a conditioned response to a CS varies as a function of the CS-US temporal interval used in training. Temporal coding theories (Barnet, Arnold, \& Miller, 1991; Desmond \& Moore, 1988; Gibbon \& Balsam, 1981; Matzel, Held, \& Miller, 1988; Miller \& Barnet, 1993) have been used to explain the response deficit observed following trace conditioning (Cole, Barnet, \& Miller, 1995), blocking (Barnet, Grahame, \& Miller, 1993), overshadowing (Blaisdell, Denniston, \& Miller, 1998), backward conditioning (Matzel et al., 
1988), simultaneous conditioning (Barnet et al., 1991), as well as the memory integration that apparently occurs following second-order conditioning and sensory preconditioning (Barnet, Cole, \& Miller, 1997). The temporal coding hypothesis put forth by Miller and his colleagues states that (a) temporal contiguity is both necessary and sufficient for learning to occur; (b) associations not only link the CS-US representation in memory, but also automatically incorporate the temporal relationship between the two events as an attribute of the learned association (i.e., subjects create temporal maps linking the events in memory); (c) the form and timing of the conditioned response is determined by this temporal map; and (d) animals can integrate temporal information (here referred to in graphical representation as temporal maps) when elements common to these maps are presented together, even when the elements were never trained together.

On the basis of the findings that subjects encode various attributes of the reinforcer in instrumental (Colwill, 1991; Colwill \& Rescorla, 1988) and Pavlovian preparations (Blaisdell, Denniston, \& Miller, 1997; Colwill \& Motzkin, 1994), and that the time at which an US is expected is encoded as an attribute of the CS-US association (Goddard, 1995; Goddard \& Jenkins, 1988), Barnet and Miller (1996) questioned whether a Pavlovian conditioned inhibitor could also provide information about the temporal location of an omitted aversive US. On the basis of the temporal coding hypothesis, Barnet and Miller hypothesized that maximal conditioned inhibition would be observed when a conditioned inhibitor predicted the absence of the US at the same temporal location as a transfer excitor predicted the presence of the US. In their conditioned lick suppression study, they factorially varied the temporal relationship of the inhibitor and the excitatory CS with which it was trained, and the temporal relationship between the training excitatory CS and the shock US. Two groups of rats received Pavlovian inhibition training composed of two reinforced and two nonreinforced trial types. The reinforced trials consisted of two Pavlovian CSs (A and B) that were trained as excitatory signals for the US. In both groups, CS A was trained as a delay CS in which the CS preceded the presentation of the US with no gap between CS termination and US onset (i.e., A $\rightarrow+$ ), while CS B was presented simultaneously with the US (i.e., B+). All CSs were $5 \mathrm{~s}$ in duration, and the US was a 5-s footshock. On the nonreinforced trials, subjects were exposed to two independent signals for US omission ( $\mathrm{X}$ and $\boldsymbol{Y}$, inhibitors). For Group Serial, $\mathrm{X}$ was established as a simultaneous inhibitor for A (i.e., $\mathrm{XA}^{-}$), and $\boldsymbol{Y}$ was established as a serial inhibitor for A (i.e., $\boldsymbol{Y} \rightarrow \mathrm{A}^{-}$). For Group Simultaneous, $\mathrm{X}$ was established as a simultaneous inhibitor for $\mathrm{B}\left(\mathrm{XB}^{-}\right)$, and $\boldsymbol{Y}$ was established as a serial inhibitor for $\mathrm{B}\left(\boldsymbol{Y} \rightarrow \mathrm{B}^{-}\right)$. At test, both $\mathrm{X}^{\prime}$ 's and $\boldsymbol{Y}^{\prime}$ 's ability to inhibit conditioned response suppression to an independently trained delay excitor $(\mathrm{C} \rightarrow+$, the transfer excitor $)$ was assessed.

The results of Barnet and Miller's (1996) study demonstrated that maximal inhibition was observed when the inhibitor predicted the absence of the US at the same time that the transfer excitor predicted the occurrence of the US. That is, conditioned inhibition was greatest for the simultaneous inhibitor $\mathrm{X}$ when it was trained with the delay excitor $\mathrm{A}$, whereas inhibitory control was maximal for the serial inhibitor $\boldsymbol{Y}$ when it was trained with the simultaneous excitor B. Their results are consistent with the temporal coding hypothesis.

The present series of studies sought to extend the findings of Barnet and Miller (1996). As previously described, Barnet and Miller factorially manipulated the temporal relationships 
between the training excitors and the US, and between the inhibitor and the training excitor. One additional variable that was held constant in Barnet and Miller's study was the temporal relationship between the transfer excitor (C) and the US. If integration of the temporal maps obtained through conditioned inhibition training and transfer excitor training modulates conditioned response suppression during summation testing, then varying the relationship between the transfer excitor and the US should also modulate the effectiveness of the inhibitor. The present research tested this prediction as a means of further testing whether conditioned inhibitors encode the temporal location of omitted USs as predicted by the temporal coding hypothesis.

In Barnet and Miller's (1996) procedure, what appeared to determine when inhibition would be observed was the temporal expectation of the omission of the US produced by the inhibitor X (or $\boldsymbol{Y}$ ) corresponding to the temporal expectation of the US based on the presentation of the transfer excitor, C. When these expectancies were the same, maximal inhibition occurred. However, if the temporal interval between the transfer excitor, $\mathrm{C}$, and the US were changed, such that the US was expected either earlier (e.g., simultaneous conditioning of $\mathrm{C}$ and the US), or later (e.g., trace conditioning of $\mathrm{C}$ ), the inhibition observed to $\mathrm{X}$ in Group Serial might not have been observed. Experiment 1 in the present series sought to test this hypothesis by manipulating the transfer excitor-US temporal relationship factorially with the training excitor-US temporal relationship, while holding constant the inhibitor-training excitor relationship. Experiment 2 factorially varied the transfer excitor-US temporal relationship and the inhibitor-training excitor relationship, while holding constant the training excitor-US temporal relationship.

\section{Experiment 1}

Experiments 1 and 2 were designed to determine if the temporal location of an omitted US controls inhibition of conditioned response suppression when the conditioned inhibitor presented in conjunction with a transfer excitor at test establishes expectancies for US omission that coincide with the time at which the US is expected based on transfer excitor training. In Experiment 1, we varied the temporal relationship between the training excitors and the US, while holding the temporal arrangement between the inhibitor and the training excitors fixed. A schematic of the temporal relationships between stimuli during training is presented in Figure 1. All experimental subjects received training that established two Pavlovian CSs as signals for US presentation. CS A was trained as a delay excitor, with CS termination coinciding with US presentation, whereas CS B was trained as a trace excitor, with CS termination preceding US onset by $5 \mathrm{~s}$. Additionally, two simultaneous conditioned inhibitors were trained as signals for US omission. Inhibitor $\mathrm{X}$ was presented simultaneously with training excitor A (i.e., XA-), and inhibitor $\boldsymbol{Y}$ was paired simultaneously with training excitor B (i.e., YB-). Before summation testing was conducted, two transfer excitors were established as excitatory signals for the US. Transfer excitor $\mathrm{C}$ was trained as a trace excitor with CS termination preceding US onset by $5 \mathrm{~s}$, whereas transfer excitor D served as a delay CS with CS termination coinciding with US onset (i.e., there was no gap between CS D termination and US onset). During summation testing, all test compounds were presented simultaneously. Control subjects were equated for the number of $\mathrm{A}$ and $\mathrm{B}$ reinforced trials, but did not receive nonreinforced exposure to $\mathrm{XA}-$ and $\mathrm{YB}^{-}$, the inhibition training trials, except during the last four days of conditioned inhibition training, during which Group Control was exposed to inhibitor $\mathrm{X}$ alone and inhibitor $\boldsymbol{Y}$ alone in order to 
reduce any external inhibition that might be induced by $\mathrm{X}$ or $\boldsymbol{Y}$ at the time of negative summation testing. Presumably, these relatively few $\mathrm{X}$ - and $\boldsymbol{Y}$ - trials were insufficient for the development of differential inhibition, an assumption that is supported by our data.

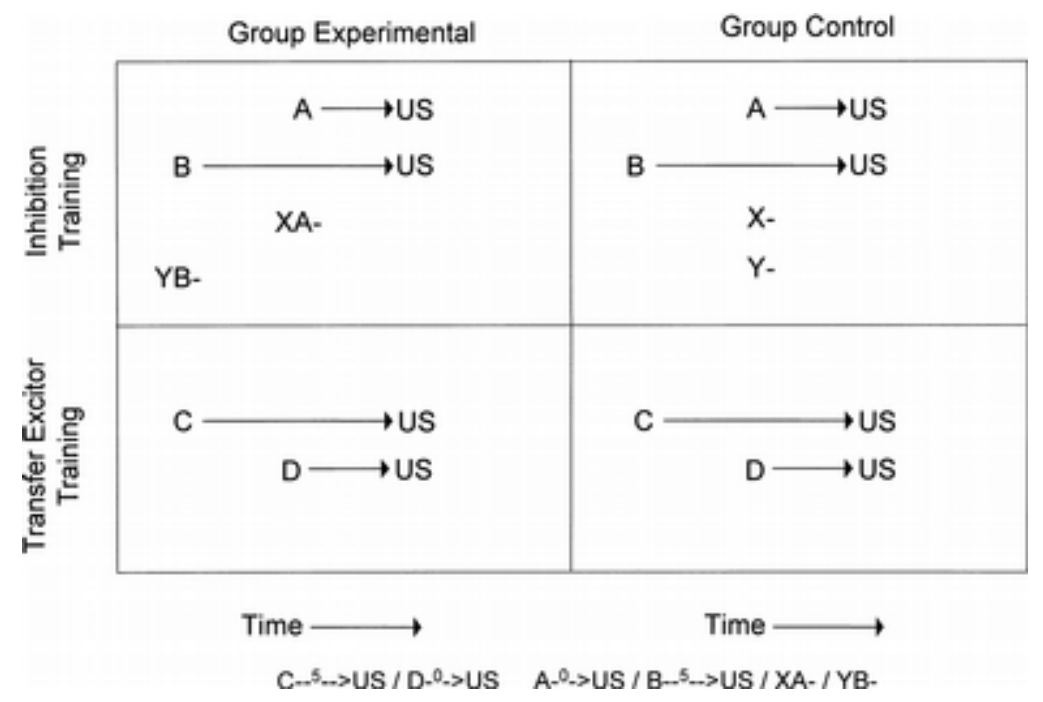

Figure 1. Schematic of temporal relationships between training stimuli in conditioned inhibition and transfer excitor training in Experiment 1 . A and $B$ represent the training excitors established as delay and trace condition stimuli, respectively. $X$ and $\boldsymbol{Y}$ represent the simultaneous inhibitors for Group Experimental, but not Group Control. $C$ and $D$ were the trace and delay transfer excitors, respectively. - represents nonreinforcement. Adjacent stimuli were presented simultaneously, and stimuli separated by arrows were presented serially. US = unconditioned stimulus

If subjects in Group Experimental do in fact encode the temporal location of the omitted US, then maximal inhibition would be expected when inhibitor $\mathrm{X}$ is tested with transfer excitor $\mathrm{D}$ and when inhibitor $\boldsymbol{Y}$ is tested with transfer excitor $\mathrm{C}$. These predictions can be derived from superimposing the temporal maps resulting from inhibition training and transfer excitor training (see Figure 2). That is, inhibitor X signals that the delay (training excitor) CS A will be nonreinforced at a specific point in time. The time at which US omission is expected based on inhibitor X coincides with the temporal location for US presentation predicted by the transfer excitor D, but not C (see Figure 2, Panels 3 and 1, respectively). Because these two expectancies, one for US omission and one for US presentation are temporally congruent, maximal inhibition would be expected. A similar analysis for inhibitor $\boldsymbol{Y}$ predicts that maximal inhibition will be observed on a simultaneous test presentation with transfer excitor C, but not D (see Figure 2, Panels 2 and 4, respectively). Notably, these predictions differ from those generated by the view that contiguity alone determines both excitatory and inhibitory strength. From that view point, delay excitors should be more strongly associated with USs than are trace excitors, and inhibitors trained with simultaneous presentation of a CS ought yield inhibitory strength that always varies directly with the strength of the excitor. 


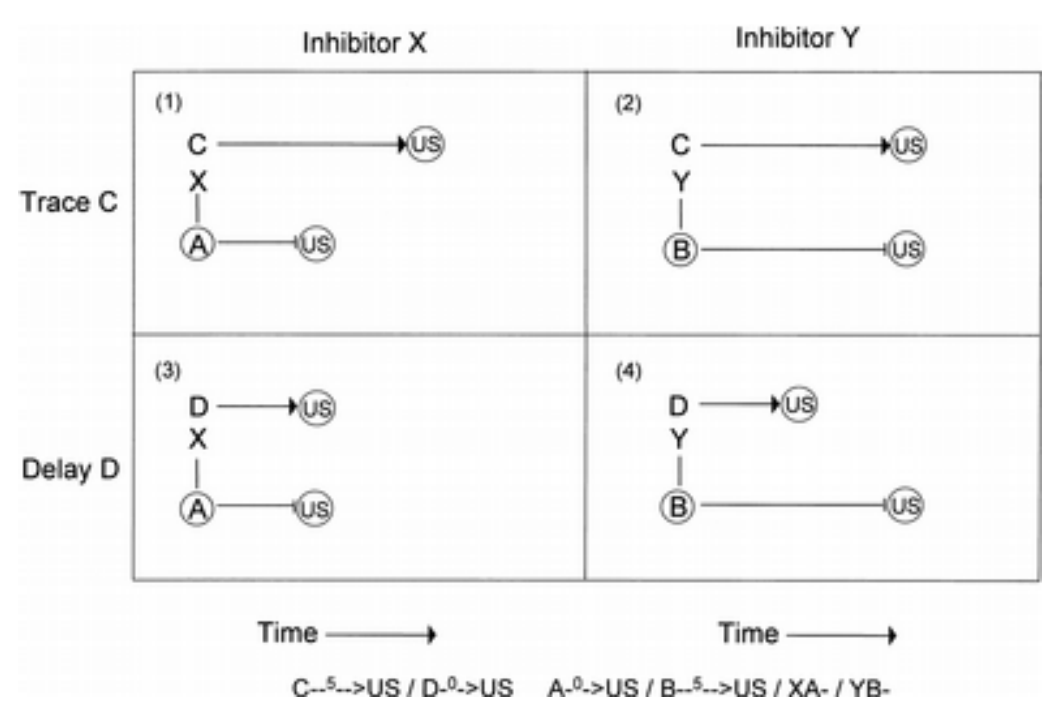

Figure 2. Hypothetical expectancies generated at test in Group Experimental, as a result of conditioned inhibition training and transfer excitor training. Horizontal lines indicate a forward expectancy. Horizontal lines with an arrow represent an expected unconditoned stimulus (US), horizontal lines ending with a cross bar indicate that an otherwise expected US will be omitted. Vertical lines indicate a simultaneous expectancy. $C, D, X$, and $\boldsymbol{Y}$ designate test stimuli; circled $A, B$, and US designate activated representations of events that are physically absent at test. Maximal inhibition is hypothesized to occur when the time at which the US is expected based on $A$ and $B$ is congruent with the time at which the US is expected to be omitted based on $X$ and $Y$. Panel 1: Test CX; Panel 2: test CY; Panel 3: test DX; Panel 4: test DY

\section{Method}

\section{Subjects}

The subjects were 24 male and 24 female, experimentally naive, Sprague-Dawley rats. Body weights ranged from 289-424 g for males and 249-317 g for females. The animals were individually housed in standard hanging, stainless-steel, wire-mesh cages in a vivarium maintained on a 16:8-hr light-dark cycle. All training occurred approximately midway through the light portion of the cycle. Subjects were allowed free access to food in their home cages, whereas access to water was gradually decreased to $10 \mathrm{~min}$ of access per day before the initiation of the experiment. Subjects were randomly assigned to two groups, $n=28$ for Group Experimental, and $n=20$ for Group Control, counterbalanced for sex. All subjects were handled for $30 \mathrm{~s}$ three times per week from weaning until the initiation of the study.

\section{Apparatus}

Two types of experimental chambers were used. Chamber $\mathrm{R}$ was rectangular in shape and measured $22.75 \times 8.25 \times 13.0 \mathrm{~cm}(1 \times \mathrm{w} \times \mathrm{h})$. The walls and ceiling of the chamber were constructed of clear Plexiglas and the floors consisted of stainless-steel rods measuring $0.48 \mathrm{~cm}$ in diameter, spaced $1.5 \mathrm{~cm}$ center-to-center. The rods were connected by NE2-neon bulbs which 
allowed for the delivery of constant-current footshock produced by a high-voltage AC circuit in series with a 1.0-M $\Omega$ resistor. Each of six copies of Chamber $\mathrm{R}$ were housed in separate sound and light attenuating environmental enclosures. Chamber $\mathrm{R}$ was dimly illuminated by a $2.0-\mathrm{W}$ (nominal at $120 \mathrm{~V} \mathrm{AC}$ ) house light driven at $60 \mathrm{~V} \mathrm{AC}$. The bulb was located on the inside wall of the environmental enclosure, approximately $30 \mathrm{~cm}$ from the center of the experimental chamber. Background noise, mostly from a ventilation fan, was $74 \mathrm{~dB}$ SPL (C).

Chamber V, was a $25.5-\mathrm{cm}$ long box in the shape of a vertical truncated-V. The chamber was 28 $\mathrm{cm}$ high, $21 \mathrm{~cm}$ wide at the top, and narrowed to $5.25 \mathrm{~cm}$ wide at the bottom. The ceiling was constructed of clear Plexiglas, the front and back walls were black Plexiglas, and the side walls were stainless steel. The floor consisted of two $25.5-\mathrm{cm}$ long parallel metal plates, each $2-\mathrm{cm}$ wide and separated by a $1.25-\mathrm{cm}$ gap. A constant-current footshock could be delivered through the metal walls and floor of the chamber. Each of six copies of Chamber V were housed in separate sound- and light-attenuating enclosures. Chamber V was illuminated by a 7-W (nominal at $120 \mathrm{~V} \mathrm{AC}$ ) bulb driven at $60 \mathrm{~V} \mathrm{AC}$. The bulb was mounted on the inside wall of the environmental enclosure, approximately $30 \mathrm{~cm}$ from the center of the experimental chamber, with the light entering the chamber primarily by reflection from the ceiling of the environmental enclosure. The light intensities in Chambers $\mathrm{R}$ and $\mathrm{V}$ were approximately equal, despite the discrepancy in the bulbs used, due to differences between the chambers in the opaqueness of the chamber walls.

Chambers $\mathrm{R}$ and $\mathrm{V}$ could each be equipped with a water-filled lick tube. When inserted, the lick tube extended about $1 \mathrm{~cm}$ into a cylindrical drinking recess that was set into one of the narrow Plexiglas walls of the chamber. Each drinking recess was left-right centered with its bottom 1.75 $\mathrm{cm}$ above the floor of the chamber. The recess was $4.5 \mathrm{~cm}$ in diameter and $5 \mathrm{~cm}$ deep. An infrared photobeam was projected horizontally across the recess, $1 \mathrm{~cm}$ in front of the lick tube. In order to drink from the lick tube, subjects had to insert their heads into the recess, thereby breaking the photobeam. Thus, we could monitor exactly when subjects were accessing the lick tube. Three speakers, mounted on separate walls in each enclosure, could deliver the following auditory cues: a 6 per s click train, a white noise, a low-frequency tone (compound of 300 and $320 \mathrm{~Hz}$ ), and a high-frequency tone (compound of 3000 and $3200 \mathrm{~Hz}$ ), each $8 \mathrm{~dB}(\mathrm{C})$ above the ambient background of $74 \mathrm{~dB}(\mathrm{C})$. Each enclosure also contained a buzzer that could produce a "buzz" $8 \mathrm{~dB}(\mathrm{C})$ above the background. Each chamber could also provide a flashing-light stimulus ( $0.17 \mathrm{~s}$ on, $0.17 \mathrm{~s}$ off). In Chamber $\mathrm{R}$, the flashing light was a $25-\mathrm{W}$ bulb (nominal at $120 \mathrm{~V} \mathrm{AC}$ ) driven at $56 \mathrm{~V} \mathrm{AC}$, whereas the flashing light in Chamber V was a 100-W bulb (nominal at $120 \mathrm{~V} \mathrm{AC}$ ) driven at $56 \mathrm{~V} \mathrm{AC}$. All CSs were $5 \mathrm{~s}$ in duration and the US was a 0.5-s, 1.0-mA footshock.

\section{Procedure}

There were three critical phases in this experiment. In the first phase, Group Experimental received conditioned inhibition training consisting of $\mathrm{A}^{0} \rightarrow+, \mathrm{B}^{5} \rightarrow+, \mathrm{XA}-$, and $\mathrm{YB}-$ pairings, in which $A$ and $B$ represent the two training excitors. The superscripts 0 and 5 represent the interval between CS termination and US onset. Thus, CS A served as a delay CS (i.e., there was 
no gap between CS termination and US onset), while CS B was established as a trace CS in which CS termination occurred $5 \mathrm{~s}$ before US onset. The two inhibitors, $\mathrm{X}$ and $\boldsymbol{Y}$, were each simultaneously paired with their respective training excitor, A or B. The second phase of the experiment established CSs C and D as signals for US presentation. CS C was trained with a 5-s gap between CS termination and US onset (i.e., $C^{5} \rightarrow+$ ), whereas CS D was trained as a delay excitor (i.e., $\mathrm{D}^{0} \rightarrow+$ ). The third phase of the experiment was a summation test with the transfer excitors, in which the potential of $\mathrm{X}$ and $\boldsymbol{Y}$ to reduce conditioned response suppression to the transfer excitors (C and D) was assessed.

Conditioned inhibition training occurred in Context CI Train, which was Chamber V for half of the subjects in each of the two groups, and Chamber $\mathrm{R}$ for the remaining subjects. Context Transfer Train was used for transfer excitor training. This context was created by switching the type of enclosure from that which was used for inhibition training. Animals that received inhibition training in Chamber V were now trained in Chamber R. Finally, summation testing occurred in Context Test, which was created by testing animals in a different example of Chamber $\mathrm{R}$ than that which was used in either inhibition or transfer excitor training. Context Test was further differentiated from Chamber $\mathrm{R}$ by the addition of a Plexiglas floor plate that covered the grid floor and an odor cue (one drop of methyl salicylate on a wooden block inside the environmental enclosure), as well as by turning off the house light that otherwise illuminated Chamber R. Different contexts were used between phases in order to ensure that an associatively neutral context was present at the time of transfer excitor training and again at testing. This ensured that any reduction in conditioned response suppression to the target cues at test could only be attributed to inhibition training, and not to contextual associations acquired during inhibition or transfer excitor training.

Following the completion of inhibition and transfer excitor training, two summation tests were conducted with each subject. During the first of these tests, one of the transfer excitors was presented either alone or in conjunction with an inhibitor, and the ability of these stimuli to disrupt ongoing drinking behavior was measured. On the next day, the second summation test was administered. Subjects were presented with the inhibitor (if any) and transfer excitor that had not been tested on the preceding day. For example, if a subject was tested for conditioned response suppression to CX on the first test day, then it could have been tested on D or DY during the second test day. Consequently, the first test was expected to have little impact on the outcome of the second test.

\section{Acclimation}

Acclimation to the experimental chambers was conducted in Context CI Train on Day 1, Context Transfer Train on Day 2, and Context Test on Day 3. During these daily 30-min sessions, waterfilled lick tubes were available and no CSs nor USs were presented. This phase of the experiment served to establish a stable baseline of drinking behavior, departure from which would serve as the dependent variable during testing.

\section{Conditioned inhibition training}


Following acclimation, the water-filled lick tubes were removed from all chambers. Inhibition training was conducted during daily 90-min sessions, on Days 4-20, and 27-29 in Context CI Train. During each of these 20 sessions, all subjects received $6 \mathrm{~A}^{0} \rightarrow+$, and $6 \mathrm{~B}^{5} \rightarrow+$ trials, and additionally, Group Experimental but not Group Control received $6 \mathrm{XA}-$ and $6 \mathrm{YB}-$ trials. The flashing light and high-frequency complex tone served as CSs A and B, counterbalanced within groups, whereas the buzzer and the white noise stimuli served as inhibitors $\mathrm{X}$ and $\boldsymbol{Y}$, counterbalanced within groups. All sessions began with a reinforced trial; the remaining reinforced and nonreinforced trials were pseudorandomly distributed within the session. The mean intertrial interval (ITI, CS onset to CS onset) was $3.5 \mathrm{~min}$ with a range of 2 to $5 \mathrm{~min}$. Group Control was equated for the number and distribution of $\mathrm{A}^{0} \rightarrow+$, and $\mathrm{B}^{5} \rightarrow+$ trials, but received no exposures to $\mathrm{X}$ and $\boldsymbol{Y}$ except during the last four sessions of inhibition training (Days 20, 27, 28, and 29), during which there were 6 exposures per session to Stimulus $X$ and Stimulus $\boldsymbol{Y}$, presented alone without reinforcement. This exposure to $\mathrm{X}$ and $\boldsymbol{Y}$ was intended to reduce any potential external inhibition by $\mathrm{X}$ and $\boldsymbol{Y}$ during testing, and yet be insufficient nonreinforced exposure to produce differential inhibition (e.g., Miller, Hallam, Hong, \& Dufore, 1991). For Group Control the white noise or buzzer served as the control inhibitor X, counterbalanced within groups.

\section{Transfer excitor training}

All subjects received transfer excitor training on Days 21-26, which consisted of 3 administrations of $\mathrm{C}^{5} \rightarrow+$, and 3 presentations of $\mathrm{D}^{0} \rightarrow+$ during each of the six daily 90 -min sessions in Context Transfer Train. The click train and low-frequency complex tone served as CSs C and D, counterbalanced within groups. Trial type (C or D) was pseudorandomly distributed within each session, and trials were separated by a 15-min mean ITI that ranged from $7.5 \mathrm{~min}$ to $22.5 \mathrm{~min}$.

\section{Reacclimation}

On Days 30 and 31, all subjects were reacclimated to Context Test during daily 30-min sessions. During these reacclimation sessions, the water-filled lick tubes were returned to the chambers in order for baseline drinking to be reestablished from any disruption produced by the footshock USs.

\section{Testing}

On Days 32 and 33, all animals were tested for conditioned suppression of ongoing drinking in the presence of the target stimuli in Context Test. No element from one test day was used on the second test day. The order of element testing across the two days was fully counterbalanced. During these test sessions, animals were allowed to drink from the lick tubes for 5 cumulative seconds, after which the target stimuli were presented. Thus, all subjects were drinking at the moment of CS onset. The time to complete an additional 5 cumulative seconds of licking in the presence of the test stimulus was recorded. A 10-min ceiling was imposed on the suppression scores. Any subject taking more than $60 \mathrm{~s}$ to complete the first five cumulative seconds of drinking (prior to CS onset), thus showing a reluctancy to drink in the test context, was scheduled to be eliminated from the data analysis. 
Group Experimental was divided into six subgroups prior to the start of the experiment, representing all six different possible pairs of test stimuli selected to have no elements in common. One subgroup $(n=4)$ was tested on excitor $C$ on one test day, and DX on the other test day, counterbalanced for order (hereafter referred to as C/DX). The remaining five subgroups were tested for conditioned response suppression to C/DY $(n=4), \mathrm{CX} / \mathrm{D}(n=4), \mathrm{CX} / \mathrm{DY}(n=6)$, CY/D $(n=4)$, and CY/DX $(n=6)$. With the counterbalancing of test order within subgroups, this produced 12 test conditions. Group Control was divided into two subgroups. One subgroup was tested on C and DX $(n=10)$, while the other was tested on CX and D $(n=10)$. Again, test order was counterbalanced within subgroups between the two test sessions. Group Control was tested on $\mathrm{X}$ but not $\boldsymbol{Y}$ because its training procedure left $\mathrm{X}$ and $\boldsymbol{Y}$ equivalent due to the counterbalancing of the physical cues serving as $\mathrm{X}$ and $\boldsymbol{Y}$. Subjects tested on compound test stimuli received the stimuli in a simultaneous test (i.e., with elements having a common onset).

\section{Data analysis}

Prior to statistical analysis, all conditioned response suppression scores were converted to log (base 10) scores, in order to permit the use of parametric statistics. An alpha level of .05 was adopted for all statistical tests in both experiments. As a result of our testing procedure, in which each animal was tested on two sets of test stimuli containing no common elements (e.g., CX and DY), the design was an incomplete factorial, in which for example a subject tested on CX on one test day could not then be tested on C, CY, or DX, on the second test day. Because each subject was tested on two orthogonal stimuli, no overall analysis of variance was performed in this experiment nor Experiment 2. Instead, a series of independent $t$ tests were conducted to test for differences in conditioned response suppression to each of the test compounds $(\mathrm{C}, \mathrm{CX}, \mathrm{CY}, \mathrm{D}$, DX, and DY) between Test Days 1 and 2. Following this analysis, the suppression scores for each test stimulus compound was pooled across test days. Analysis of the conditioned response suppression scores pooled over test days was accomplished with a series of one-way betweensubject analysis of covariances (ANCOVAs) in which each statistical test contained only one data point from each subject. The subjects' gender and the physical stimulus serving as the transfer excitor (click or tone) were used as covariates in all statistical analyses in both experiments. For example, the data pooled over test days for $\mathrm{C}, \mathrm{CX}$, and $\mathrm{CY}$ contained independent measures from different subjects. Finally, planned comparisons of the group adjusted means were conducted using the adjusted error term from the overall ANCOVA.

Prior to the completion of this experiment, one animal from Group Experimental that was scheduled to be tested on D and CY, Test Days 1 and 2 respectively, died before the completion of the experiment. Two animals from Group Control required greater than $60 \mathrm{~s}$ to complete the 5 cumulative seconds of drinking prior to the presentation of the test stimuli, thus showing a reluctancy to drink in Context Test. The data from both test sessions was discarded for these subjects. The animals from Group Control were to have been tested on DX/C and D/CX (Test Day 1/Test Day 2). Collapsed over test day, this left $n \mathrm{~s}=9$ for each test stimulus in Group Control. 


\section{Results and Discussion}

The central findings from this study were that the greatest conditioned inhibition was observed when the expectation of no-US evoked by $\mathrm{X}$ and $\boldsymbol{Y}$ corresponded temporally to the expectation of the US evoked by $\mathrm{C}$ and $\mathrm{D}$. That is, reduced conditioned response suppression (i.e., behavior indicative of greater conditioned inhibition) was observed in experimental subjects tested on CY but not $\mathrm{CX}$, relative to those tested on the transfer excitor $\mathrm{C}$ alone. Moreover, experimental animals tested on either DX or DY displayed less conditioned response suppression (i.e., more inhibition) than those tested on the transfer excitor D alone, although the amount of conditioned response suppression observed in animals tested on DY was more than that observed in animals tested on DX. No significant differences in conditioned response suppression (i.e., no conditioned inhibition) were obtained in control subjects tested on the CX and DX stimulus compounds relative to those tested on $\mathrm{C}$ and $\mathrm{D}$ alone. Figure 3 depicts the adjusted mean $\log$ time to complete the 5 cumulative seconds of drinking in the presence of the test stimuli.

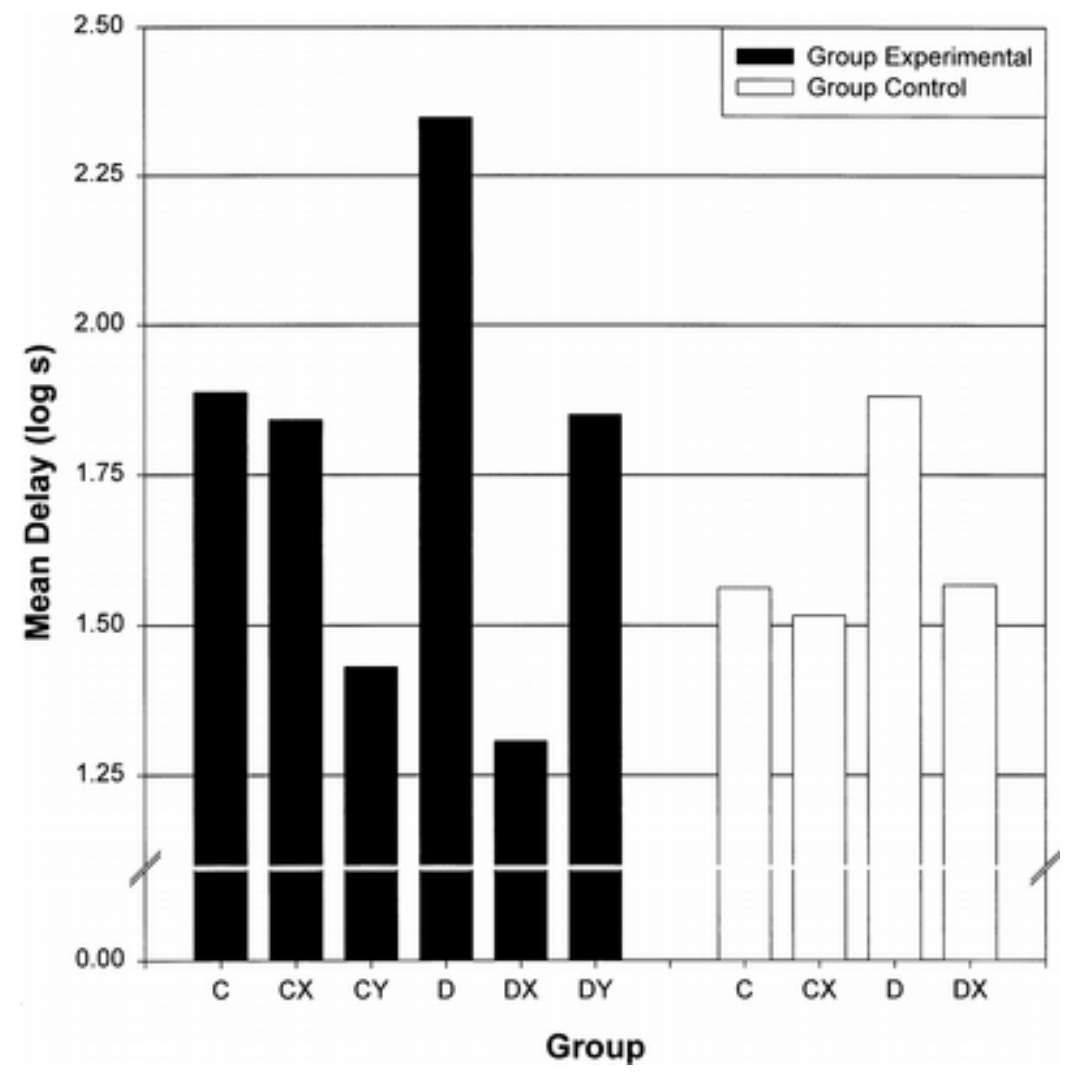

Figure 3. Experiment 1. Adjusted mean times to complete 5 cumulative seconds of drinking in the presence of the test stimuli. Greater scores indicate less conditioned inhibition

These findings were confirmed with statistical analysis. Prior to analyzing conditioned suppression to the CSs, we first looked for group differences in reluctance to drink in the test context in the absence of any CS. Any observed difference in baseline drinking would suggest a 
potential confound. Comparisons of the pre-CS times to complete 5 cumulative seconds of drinking in the test context found no differences in baseline drinking behavior; that is, no differences in reluctance to drink in the test context was evident between subjects tested on the different test stimuli in this experiment, as well as in Experiment 2, all $F \mathrm{~s}<2.16$, $p \mathrm{~s}>.13$.

Having found no differences between groups in baseline drinking, we turned to the suppression scores in the presence of the test CSs. The series of independent $t$ tests conducted to test for differences in conditioned response suppression to each of the test compounds $(\mathrm{C}, \mathrm{CX}, \mathrm{CY}, \mathrm{D}$, DX, and DY) revealed no significant differences in the level of conditioned response suppression between test days for the same test stimuli for Group Experimental, all $t \mathrm{~s}(5-8)<1.01, p \mathrm{~s}>.33$. The series of independent $t$ tests conducted on the conditioned response suppression scores from subjects in Group Control that were tested on C, CX, D, and DX revealed no differences between test days for subjects tested on $\mathrm{C}, \mathrm{CX}$, and $\mathrm{DX}, t \mathrm{~s}(7)<1.07, p \mathrm{~s}>.32$; however, there was a significant difference between test days for subjects tested on $\mathrm{D}, t(7)=2.17, p<.05$. This difference between test days resulted from less conditioned response suppression to $\mathrm{D}$ on the second test session, relative to the first test session. Because this difference in levels of conditioned response suppression between test days for subjects tested on D was small, we pooled the conditioned response suppression scores across test days to facilitate further statistical analysis.

A one-way ANCOVA with test stimuli as the independent factor was conducted on the scores for conditioned response suppression to Stimulus $\mathrm{C}$ and its compounds for subjects in Group Experimental. The ANCOVA revealed a marginally significant difference between test stimuli, $F(2,22)=2.90, M S E=0.192, p<.08$. Planned comparisons using the adjusted error term from the ANCOVA did not reveal any loss of conditioned response suppression in subjects presented with $\mathrm{CX}$ at test relative to those tested on $\mathrm{C}$ alone, $F(1,22)<1$, whereas animals tested on $\mathrm{CY}$ did show less conditioned response suppression (i.e., conditioned inhibition) relative to those tested on $\mathrm{C}$ alone, $F(1,22)=4.56, p<.05$. The critical difference between $\mathrm{CX}$ and $\mathrm{CY}$ was marginally significant, $F(1,22)=4.12, p<.06$. A second one-way ANCOVA with test stimuli as the independent factor was conducted on the scores for conditioned response suppression to Stimulus D and its compounds for subjects in Group Experimental. The ANCOVA revealed a main effect of test stimulus, $F(2,22)=20.56, M S E=0.110, p<.001$. Planned comparisons found differences between $\mathrm{D}$ and DX, $F(1,22)=40.34, p<.001$, and $\mathrm{D}$ and $\mathrm{DY}, F(1,22)=$ $9.24, p<.01$. That significant inhibition was observed in animals tested on DY relative to D, is somewhat problematic. However, this observation is not particularly damaging because in the critical comparison the inhibition evident on the DX test was much greater than that evident on the DY test, $F(1,22)=13.40, p<.01$. Furthermore, the Temporal Coding Hypothesis does not predict that the temporal variables between stimuli will determine whether conditioned inhibition will be observed in an all-or-none fashion; rather, the temporal relationships should act in a graded fashion as the difference in temporal expectations of the US occurring and the US not occurring become more similar. Moreover, temporal similarity is presumably only one of several attributional dimensions of the US expectation that must not match in order to eliminate all interaction of the two expectations.

Analysis of Group Control's data was also accomplished through two one-way ANCOVAs with test stimulus as the factor. The ANCOVAs conducted on the conditioned response suppression 
scores for subjects tested on $\mathrm{C}$ and $\mathrm{CX}$, and on D and DX failed to reveal a difference between test stimuli, $F \mathrm{~s}(1,14)<1.07, M S E=0.415, p>.31$.

The lesser conditioned response suppression (i.e., greater inhibition) observed in experimental animals tested on CY and DX supports the view that conditioned inhibition will be maximal when the time at which the US is expected, based on the transfer excitor, is the same as the time at which the omission of the US is expected, based on the inhibitor. Figure 2 presents a hypothetical temporal map of the expectancies generated by the inhibitors and the transfer excitors. As can be seen in this diagram, only CY and DX create expectancies that are aligned in time. When the expectancies occur at inconsistent points in time (e.g., CX and DY), less inhibition is anticipated. The results from this experiment are generally consistent with these expectations.

Our use of different contexts for each phase of the experiment in order to ensure associatively neutral contexts at the time of conditioned inhibition training, transfer excitor training, and testing raises several questions. First, some animals in the present experiment (as well as in Experiment 2) showed a reluctancy to drink in context test. Therefore, one might question whether our context changes were effective. Although we are unable to directly evaluate the efficacy of our context manipulations, comparison of the log times to complete the first five cumulative seconds of drinking in the test context during acclimation (Day 3) and reacclimation (Days 30 and 31) revealed no differences between the acclimation (Day 3) and the first reacclimation session (Day 30). However by the second reacclimation session (Day 31) there was a significant reduction in the time to complete five cumulative seconds of drinking in the context during the second reacclimation session relative to the first acclimation session (Day 3 ), $F(1,43)=42.48, p<.001$. This pattern of results suggests that although animals showed a decrease in their time to complete five cumulative seconds of drinking over subsequent reacclimation sessions, suggesting that there was some reluctance to drink in the test context during reacclimation, this reluctance was no greater than that during the initial acclimation session which was given prior to any CS or US presentations. The same pattern of results was observed in Experiment 2.

Another issue raised by our use of different contexts for each phase of training and testing concerns the context specificity of conditioned inhibition. Bouton and Nelson (1994) found that conditioned inhibition can be context specific. Specifically, in their experiments they found that the target of conditioned inhibition training loses its ability to be inhibited when tested in a context other than that in which it was trained (Experiments 3 and 4), while the feature (the inhibitor) readily transfers to other contexts (Experiments 2 and 4). Bouton and Nelson's findings are entirely consistent with the present findings in that we found that a conditioned inhibitor was able to reduce responding to an independently trained transfer excitor in a different context than that used either for training the inhibitor or training the transfer excitor, provided the temporal expectations for US presentation and omission were congruent. Presumably, our observation of transfer of inhibitory control to a different context stems in part from the transfer excitor not having been inhibited during training. On the basis of Bouton and Nelson's findings, had we tested the potential of the inhibitors to transfer inhibitory control to the training excitors which had been inhibited (e.g., A and B), we might not have observed inhibition even when the 
temporal expectancies for reinforcement and nonreinforcement were temporally congruent. However, this possibility remains to be evaluated within our procedure.

Experiment 2

The purpose of Experiment 2 was to extend the results of Experiment 1 by manipulating the temporal relationships between the transfer excitors and the US, and between the inhibitors and their training excitors, while holding constant the training excitor-US temporal relationship. The primary difference between this experiment and Experiment 1 was that, rather than both inhibitors being paired simultaneously with their respective training excitor as in Experiment 1, one inhibitor $(\mathrm{X})$ was now trained as a serial inhibitor, while the other inhibitor $(\boldsymbol{Y})$ remained a simultaneous signal for US omission. Because the temporal variables for the training excitors were held constant with no gap between CS termination and US onset, only one training excitor was used (i.e., $\mathrm{A}^{0} \rightarrow+$ ). Transfer excitor training remained the same as in Experiment 1 (i.e., $\mathrm{C}^{5}$ $\rightarrow+$ and $\mathrm{D}^{0} \rightarrow+$ ). Additionally, Group Experimental received $\mathrm{A}^{0} \rightarrow+, \mathrm{X}^{0} \rightarrow \mathrm{A}-, \mathrm{YA}-$ presentations, while Group Control only received $\mathrm{A}^{0} \rightarrow+$ training and no exposure to the inhibitors except during the last 6 days of inhibition training, during which nonreinforced exposures to $\mathrm{X}$ and $\boldsymbol{Y}$ (in the absence of A) were given. A schematic of the temporal relationships between stimuli during training is presented in Figure 4.

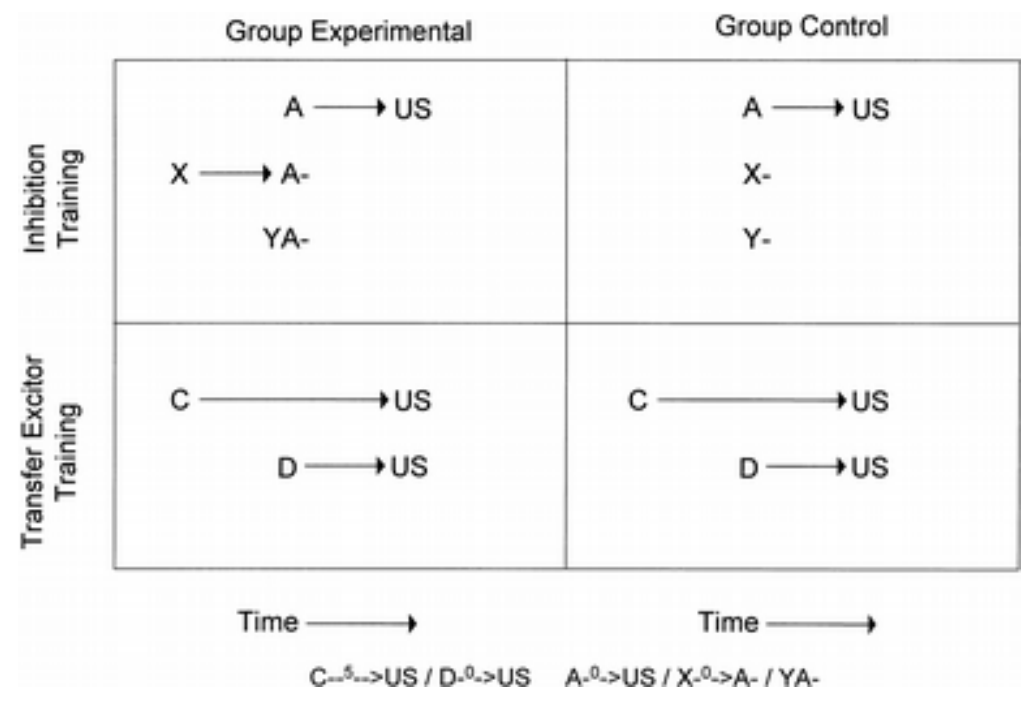

Figure 4. Schematic of temporal relationships between training stimuli in conditioned inhibition and transfer excitor training in Experiment 2. A represents the delay training excitor, and $X$ and $\boldsymbol{Y}$ represent the serial and simultaneous inhibitors, respectively, for Group Experimental, but not Group Control. $C$ and $D$ were the trace and delay transfer excitors, respectively. - represents nonreinforcement. Adjacent stimuli were presented simultaneously, and stimuli separated by arrows were presented serially. US = unconditioned stimulus

Based on the putative expectancies generated as a result of inhibition and transfer excitor training, the superimposed temporal maps (see Figure 5) predict that maximal conditioned inhibition will be observed for subjects tested on CX and DY, but not DX and CY. For example, inhibitor $\mathrm{X}$ activates an expectancy of nonreinforcement based on its nonreinforced presentation 
in serial compound with A. Because $\mathrm{X}$ and $\mathrm{A}$ (both $5 \mathrm{~s}$ in duration) were trained serially with no gap between X termination and A onset and no gap between A termination and US onset, the US would be expected to be omitted $10 \mathrm{~s}$ following the onset of $\mathrm{X}$. Thus, only transfer excitor $\mathrm{C}$ (the trace excitor with a 5-s gap between C termination and US onset) should evoke an expectancy of US presentation at the same point in time (see Figure 5, Panel 1). A similar analysis reveals that maximal inhibition (i.e., minimal conditioned response suppression) ought to be observed to DY (Panel 4), but not to DX and CY (Panels 3 and 2, respectively).

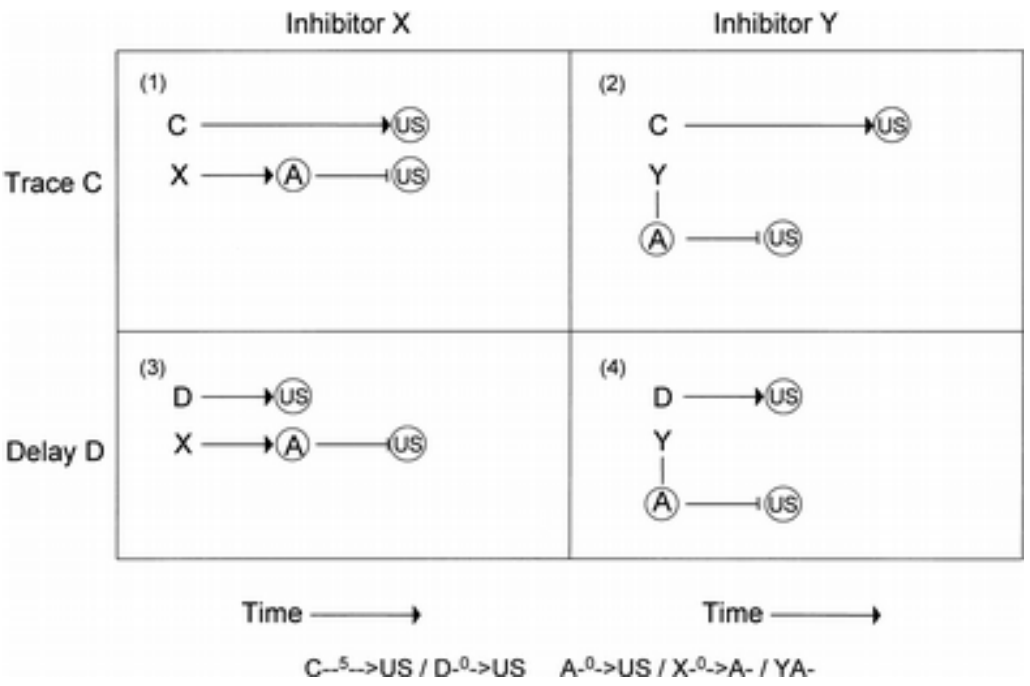

Figure 5. Hypothetical expectancies generated at test in Group Experimental, as a result of conditioned inhibition training and transfer excitor training. Horizontal lines indicate a forward expectancy. Horizontal lines with an arrow represent an expected US, horizontal lines ending with a cross bar indicate that an otherwise expected unconditioned stimulus (US) will be omitted. Vertical lines indicate a simultaneous expectancy. $C, D, X$, and $\boldsymbol{Y}$ designate test stimuli; circled $A$ and US designate activated representations of events that are physically absent at test. Maximal inhibition is hypothesized to occur when the time at which the US is expected based on $A$ is congruent with the time at which the US is expected to be omitted based on $X$ and $Y$. Panel 1: Test CX; Panel 2: test CY; Panel 3: test DX; Panel 4: test DY

\section{Method}

\section{Subjects}

The subjects were 24 male and 24 female naive Sprague-Dawley rats. Body weights ranged from 203-327 g for males and 178-248 g for females. Animal care and deprivation were the same as in Experiment 1. The subjects were divided into two groups, Group Experimental $(n=28)$, and Group Control $(n=20)$, counterbalanced within group for sex.

\section{Apparatus}


The apparatus was the same as that in Experiment 1, except the high-frequency complex tone was not used in this experiment.

\section{Procedure}

Experiment 2 was similar in design to Experiment 1. Once again, the experiment was composed of three critical phases. Group Experimental was exposed to conditioned inhibition training, in which one training excitor was established as a delay CS (i.e., $\mathrm{A}^{0} \rightarrow+$ ). Because the training excitor-US temporal interval was not manipulated in this experiment, the second training excitor (B) was omitted. As in Experiment 1, two inhibitors were trained, but now one was established as a serial inhibitor (i.e., $\mathrm{X}^{0} \rightarrow \mathrm{A}^{-}$) and the other simultaneous (YA-). Transfer excitor training (C and D) was the same as in Experiment 1.

\section{Acclimation}

Acclimation to the three contexts was the same as in Experiment 1.

\section{Conditioned inhibition training}

Conditioned inhibition training was conducted over 20 sessions, Days 4-20 and 27-29 in Context CI Train. During these daily 90-min sessions, subjects in Group Experimental received 8 $\mathrm{A}^{0} \rightarrow+, 8 \mathrm{X}^{0} \rightarrow \mathrm{A}-$, and $8 \mathrm{YA}-$ trials per session. Group Control received $8 \mathrm{~A}^{0} \rightarrow+$ trials, but lacked the critical inhibition training. Instead, these animals were merely exposed to the putative inhibitors during the last six sessions of inhibition training (Days 18-20, and 27-29); specifically, $\mathrm{X}$ and $\boldsymbol{Y}$ were presented alone, each nonreinforced eight times per session. CS A was the flashing light, and $\mathrm{X}$ and $\boldsymbol{Y}$ were the buzzer and white noise, counterbalanced within groups. Trial spacing and distribution were the same as in Experiment 1.

\section{Transfer excitor training}

Transfer excitor training (Days 21-26) was the same as in Experiment 1.

\section{Reacclimation}

Reacclimation (Days 30-32) to Context Test, was the same as in Experiment 1, except that an additional session of reacclimation was given due to several animals showing a reluctancy to drink in Context Test.

\section{Testing}

Testing (Days 33 and 34) was conducted in the same manner as in Experiment 1. Group Experimental was divided into six subgroups that were defined by all possible pairs of test stimuli selected such that no pairs shared common elements: C/DX $(n=4), \mathrm{C} / \mathrm{DY}(n=4), \mathrm{CX} / \mathrm{D}$ $(n=4), \mathrm{CX} / \mathrm{DY}(n=6), \mathrm{CY} / \mathrm{D}(n=4)$, and CY/DX $(n=6)$, where the first stimulus was tested on one of the two test days, and the second stimulus was tested on the other test day, test order being counterbalanced within groups. Group Control was similarly divided into two subgroups, 
C/DX $(n=10)$ and D/CX $(n=10)$, in which order of test was similarly counterbalanced across days. As in Experiment 1, all compound test stimuli were presented with simultaneous onsets.

\section{Data analysis}

Statistical analyses were conducted as in Experiment 1.

Five animals, three from Group Experimental and two from Group Control required greater than $60 \mathrm{~s}$ to complete the 5 cumulative seconds of drinking prior to the presentation of the test stimuli, thus showing a reluctancy to drink in Context Test. The data from both test days were discarded for these subjects. The three animals from Group Experimental were to have been tested on CX/D, CY/DX, and DX/CY (Test Day 1/Test Day 2). Collapsed over test day, this left $n=8$ for subjects tested on $\mathrm{C}, 9$ for $\mathrm{CX}, 8$ for $\mathrm{CY}, 7$ for $\mathrm{D}, 8$ for $\mathrm{DX}$, and 10 for DY. The two subjects from Group Control were to have been tested on DX/C and D/CX, leaving $n \mathrm{~s}=9$ for each test stimulus.

\section{Results and Discussion}

The central findings from this study were that experimental subjects tested on the CX and DY test compounds displayed less conditioned response suppression (i.e., behavior indicative of greater conditioned inhibition) than did subjects tested on CY and DX. As in Experiment 1, the greatest conditioned inhibition was observed when the expectation of no-US evoked by $\mathrm{X}$ and $\boldsymbol{Y}$ corresponded temporally to the expectation of the US evoked by C and D. Figure 6 depicts the adjusted mean log time to complete 5 cumulative seconds of drinking in the presence of the test stimuli. 


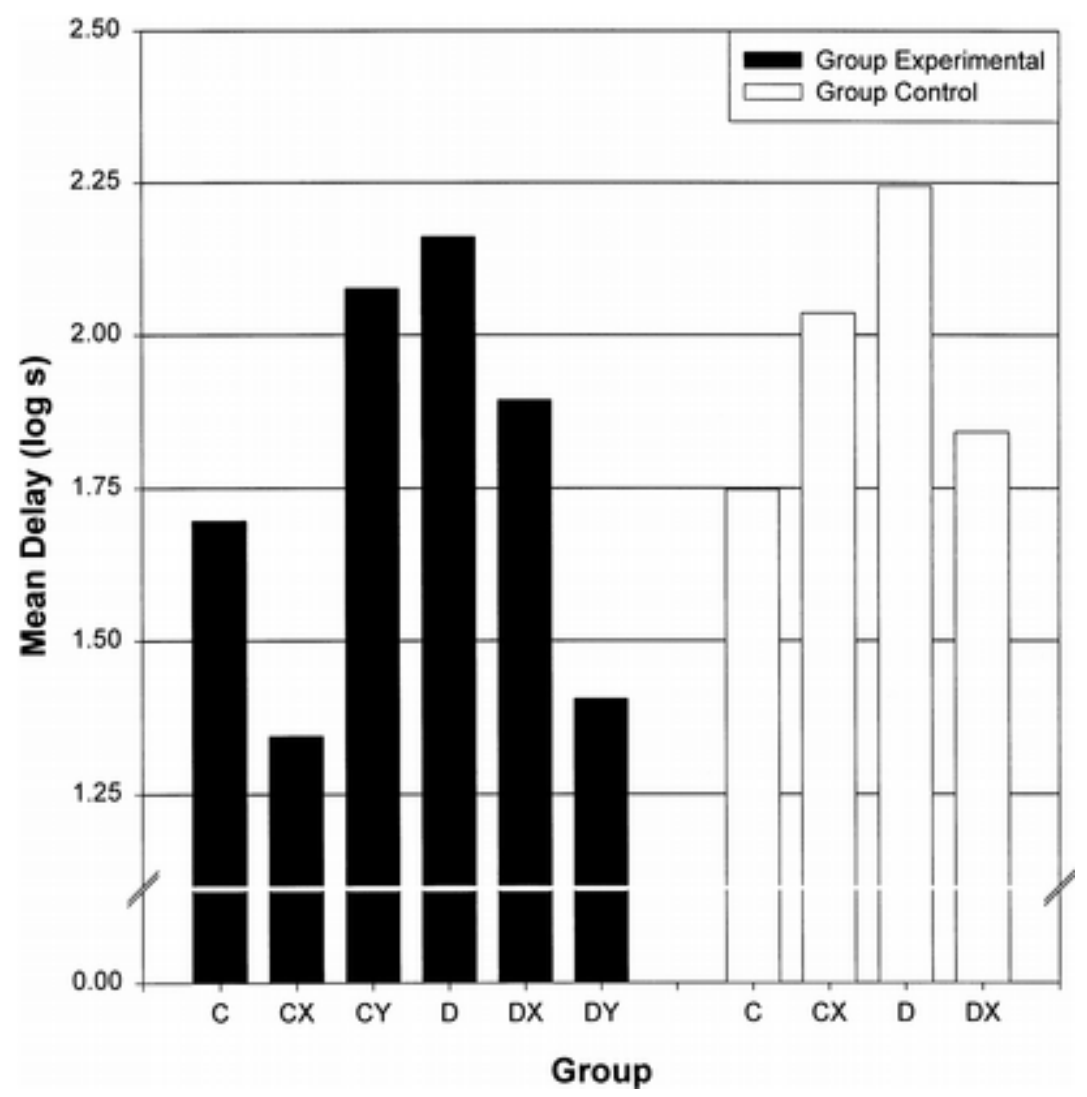

Figure 6. Experiment 2. Adjusted mean times to complete 5 cumulative seconds of drinking in the presence of the test stimuli. Greater scores indicate less conditioned inhibition

Statistical analysis confirmed the above findings. As in Experiment 1, a series of independent $t$ tests for each test stimulus was conducted to determine whether there were any differences in conditioned response suppression between test days. The $t$ tests revealed no difference in conditioned response suppression to any of the test stimuli between days, all $t \mathrm{~s}(5-8)<1$, except for the difference between days for animals tested on DX, $t(6)=2.07, p>.08$. A similar analysis for subjects in Group Control revealed no differences in levels of conditioned response suppression to each of the test stimuli between days, all $t \mathrm{~s}(7)<1.55, p \mathrm{~s}>.16$. Again, to facilitate statistical analysis, all data within groups were collapsed across test days.

The data were further analyzed with the same covariates as were used in Experiment 1. A series of one-way between subject ANCOVAs and planned comparisons were conducted to evaluate differences in conditioned response suppression between critical stimuli. For Group Experimental, the ANCOVA on stimuli C, CX, and CY found a main effect of stimulus, $F(2,20)$ $=4.78, M S E=0.236, p<.05$. Planned comparisons using the adjusted error term from the ANCOVA revealed that the differences between $\mathrm{C}$ and $\mathrm{CX}$, and between $\mathrm{C}$ and $\mathrm{CY}$ fell short of significance, $F_{\mathrm{S}}(1,20)=2.17, p<.16$, and 2.40, $p<.14$, respectively. However, in the critical comparison, less conditioned response suppression was observed to $\mathrm{CX}$ than to $\mathrm{CY}, F(1,20)=$ $9.56, p<.01$. Analysis of the conditioned response suppression by experimental subjects tested on D, DX, and DY revealed a main effect of test stimulus, $F(2,20)=6.29, M S E=0.199, p<.01$. 
Comparisons of the relative levels of conditioned response suppression observed to DX and DY to D alone, revealed that only animals tested on DY demonstrated a significant loss of suppression (i.e., inhibition), $F \mathrm{~s}(1,20)=1.32, p>.26$, and $11.69, p<.01$, respectively. More important, in the critical comparison, animals tested on DY showed less conditioned response suppression than those tested on DX, $F(1,20)=5.31, p<.05$.

Analysis of the data from Group Control was also accomplished through a series of ANCOVAs that found the differences between $\mathrm{C}$ and $\mathrm{CX}$, and between $\mathrm{D}$ and $\mathrm{DX}$ to be nonsignificant, $F \mathrm{~s}(1$, $14)=1.92, M S E=0.191, p>.18$, and 3.45, MSE $=0.208, p>.08$, respectively.

The results from Group Experimental lend support to an interpretation of temporal coding within condition inhibition that is consistent with the temporal coding hypothesis. That is, the greatest amounts of inhibition were observed when the time at which the US was expected to occur, signaled by the transfer excitor, coincided with the time at which the US was expected to be absent, conveyed by the inhibitor as a result of the training excitor with which it had been paired. Inspection of Figure 5, which depicts the hypothetical temporal maps generated as a result of transfer excitor and conditioned inhibition training, illustrates that the greatest levels of inhibition ought to be observed in subjects tested on the CX and DY stimulus compounds (Panels 1 and 4, respectively). The results of this study are largely congruent with the predictions derived from the integration of temporal maps.

One problematic finding for the temporal coding hypothesis explanation of the current data was the failure to observe a significant difference between experimental animals tested on C and CX. This lack of a difference might have been due to the relatively low levels of conditioned response suppression observed to the trace excitor $\mathrm{C}$, in conjunction with a floor effect imposed on conditioned response suppression to CX. Alternatively, the work of Holland and his colleagues (Holland, 1989; Holland \& Lamarre, 1984; Lamarre \& Holland, 1987) provides a different explanation for this result. Collectively, their studies have found that serial inhibitors do not transfer to independently trained excitors as readily as do simultaneous inhibitors. Instead, transfer is strongest when the target is a stimulus that was trained as an excitor in another feature-negative problem. However, such an explanation seems inapplicable here because the difference between $\mathrm{CX}$ and $\mathrm{CY}$ was significant. While this result might have stemmed from increased conditioned response suppression to $\mathrm{C}$ when $\boldsymbol{Y}$ was compounded with the transfer excitor, this too seems implausible in light of the cross-over nature of the results; that is, conditioned response suppression to CX was less than that observed to CY, whereas conditioned response suppression to DY was less than that observed to DX. This suggests that $\mathrm{X}$ produced more inhibition when it was presented in compound with $\mathrm{C}$ than when it was presented in compound with D (if any), and $\boldsymbol{Y}$ produced more inhibition when tested with D than when it was tested with $\mathrm{C}$ (if any).

General Discussion

The present experiments investigated the role of temporal variables in the transfer of conditioned inhibition. Experiment 1 demonstrated that simultaneous inhibitors transfer their behavioral control to an independently trained excitor depending on the temporal relationships between the training excitor and the US and between the transfer excitor and the US. Specifically, transfer of 
inhibitory control was maximal when the inhibitor was paired with a training excitor that predicted the absence of the US at the same point in time as the transfer excitor predicted the occurrence of the US. Experiment 2 extended these findings by varying the temporal intervals between the two conditioned inhibitors and the training excitor as well as the transfer excitors and the US, while holding the training excitor-US interval constant. Again, inhibition was greatest when the time at which the US was omitted (signaled by the inhibitor and the training excitor) was consistent with the time at which the US was expected (based on the transfer excitor).

These findings lend support to the temporal coding hypothesis (Barnet et al., 1991; Blaisdell et al., 1996; Cole et al., 1995; Matzel et al., 1988; Miller \& Barnet, 1993). As previously stated, the temporal coding hypothesis posits that (1) temporal contiguity is both necessary and sufficient for learning to occur; (2) associations encode the temporal relationships between associates; (3) encoded temporal information influences the nature and timing of a conditioned response; (4) Subjects integrate temporal maps that contain common elements.

This series of studies evaluated Tenets 2,3 , and 4 of the temporal coding hypothesis. In order to obtain the observed patterns of results from Experiments 1 and 2, the subjects must have encoded the time at which the US was presented following the transfer excitor (trace vs. delay in both experiments) as well as the training excitor-US temporal relationships (trace vs. delay in Experiment 1, and delay in Experiment 2). Additionally, associations to US omission (the inhibitors) must encode the time at which US omission is expected, based conjointly on the inhibitors' relationships to the training excitors and the training excitor-US temporal relationship. At test, there was evidence that subjects integrated their temporal expectancies created through both inhibition and transfer excitor training. Finally and importantly, the nature of this temporal integration determines the form of the conditioned response, namely, whether it will be excitatory or inhibitory in the current situation (see Miller \& Barnet, 1993, for elaboration).

Recent neural network models that address timing of conditioned responding (Desmond \& Moore, 1988) and occasion setting (Schmajuk, Lamoureux, \& Holland, 1998) have also been used to explain conditioned inhibition and the ability of occasion setters and conditioned inhibitors to transfer their inhibitory control. The connectionist model presented by Desmond and Moore, relies on the temporal relationships between CSs and USs to determine the magnitude of conditioned responding. In their model, CS onset and termination activates an "onset process" and an "offset process." These onset and offset processes each activate a series of trace elements, with additional elements being activated during each "time step" of the CS (i.e., "onset traces") and an equivalent number of elements being activated over time following termination of the CS (i.e., "offset traces"). These elements are maximally eligible for association with the US at the time of trace initiation, and then decay geometrically. If a US is presented while these traces are active, then conditioned responding to the most eligible trace will be strengthened at the expense of less eligible traces. Similarly, if a US is omitted while the traces are active, then inhibition to the most active trace will be accrued. Applied to the present series of experiments, if a CS A is paired with a US on some trials, A will produce conditioned responding; however, on nonreinforced trials in which CS A is compounded with a CS X (e.g., $\mathrm{AX}$-noUS), the traces activated by $\mathrm{X}$ when the US was expected but not delivered will accrue 
inhibition. During summation testing, if the inhibitory traces are superimposed on a conditioned response tendency elicited by another excitor (a transfer excitor), negative summation will be observed. Thus, according to Desmond and Moore, transfer of conditioned inhibition is dependent on simultaneous activation of a CR tendency and the inhibitory trace activated by the conditioned inhibitor, a position fully consistent with the present results and the temporal coding hypothesis.

A more recent neural network model put forth by Schmajuk, Lamoureux, and Holland (1998), distinguishes between the ability of simultaneous and serial negative features to transfer inhibitory control to other stimuli. In their model, a simultaneous negative feature gains direct inhibitory control over the $\mathrm{CR}$ as a result of its close temporal proximity to A, and is thus able to inhibit responding to any excitatory CS. In contrast, serial negative features inhibit responding to their target CSs through hidden units in the model. This difference in the locus of the inhibitory association results from the stimulus trace of the serial negative feature being relatively small at the time of target presentation. The potential of the serial negative feature to inhibit responding results from inhibition by a hidden unit which is activated by both the feature and the target. Activation of these hidden units reduces the strength of the conditioned response. Therefore, transfer of inhibitory control is limited by the potential of the feature and the target to access the hidden units. That is, an independently trained excitor is less effective at gaining access to the hidden units than the target CS of training, resulting in little transfer of inhibitory control. However, a feature tested with a target CS from another feature-negative discrimination should readily transfer inhibitory control, because both the feature and the target CS have access to the hidden inhibitory units. Applied to the present series of experiments, Schmajuk et al.'s model would predict transfer of inhibition for the simultaneous, but not serial inhibitors. At present, there are no published simulations of their model that address whether the temporal relationship between the transfer excitor and the US would affect the amount of transfer of inhibition by a simultaneous inhibitor. In contrast to their model, the present experiments found transfer of inhibitory control by both serial and simultaneous inhibitors that was dependent on the inhibitor and the transfer excitor activating simultaneous expectations for US omission and US presentation, respectively.

The present results suggest a discrepancy with other reports in the literature. Holland and Lamarre (1984, Experiment 2) trained rats on two discrimination problems, one of which was a simultaneous feature-negative discrimination, and the other was a serial feature-negative discrimination, and then assessed the ability of the negative features to transfer their inhibitory behavioral control to various target CSs. Their results found that a serial feature inhibited responding to a target CS that had participated in feature-negative training when tested serially or simultaneously, but less transfer was observed when it was tested with a target CS that had participated in a simultaneous feature-negative discrimination. The ability of a simultaneous feature to transfer to a target CS was more ambiguous. The simultaneous feature transferred to a target CS from a serial feature-negative discrimination when it was tested either simultaneously or serially with the target CS. However, when the simultaneous feature was tested with its own target CS, reduced responding was found during a simultaneous test, but not a serial test, a result consistent with the temporal coding hypothesis. In their Experiment 3, Holland and Lamarre again found that following simultaneous and serial feature-negative training, transfer of inhibition was greater with simultaneous features than serial features, however their results 
depended on the manner of testing. When the serial feature was tested with an excitor, the feature failed to modulate responding to the excitor regardless of whether the feature-excitor temporal relationship was serial or simultaneous. But, when the simultaneous feature was tested with the excitor, transfer was observed with simultaneous but not serial tests, a result consistent with the temporal coding hypothesis, which predicts that the greatest inhibition should be observed when the feature and the target activate expectations for US presentation and US omission at a common time. In both Experiments 2 and 3, Holland and Lamarre found that a simultaneous feature failed to modulate responding to an excitor when the feature was tested serially, which according to the temporal coding hypothesis would activate expectations of the US and no-US occurring at different times, and consequently result in reduced transfer of inhibitory control.

A number of procedural differences exist between Holland and Lamarre's (1984) study and the present investigations. First, their preparation used CSs and features that were of considerably longer duration (1-min CSs). Second, the present study used transfer excitors that were trained independently in a different context than that in which inhibition training was given, whereas Holland and Lamarre used a single context for these purposes. More recently, Holland and Lamarre's findings have been replicated by Lamarre and Holland (1987) and Holland (1991). Importantly, Holland (1991) used CSs of shorter duration (5 s), but with a 5-s gap between termination of the feature and onset of the target CS, whereas the present series of experiments used no gap between the serial inhibitor and the excitor. Additionally, Holland also used an appetitive US in contrast to our aversive US. Perhaps due to these differences in the present research, but for whatever reason, we obtained transfer of inhibition with serial inhibitors, which depended on the temporal relationship of the transfer excitor and the US. For instance, in Experiment 2 the serial inhibitor $\mathrm{X}$ produced transfer only when it was presented with the trace transfer excitor (C), which produced an expectancy of US presentation corresponding to the time at which omission of the US was anticipated based on the inhibitor (see Figure 5, Panel 1). When the same serial inhibitor was tested with the delay excitor (D), no transfer was observed (Figure 5, Panel 3).

Barnet and Miller (1996) also found that a serial inhibitor could best transfer to an independently trained excitor when the temporal arrangement of the inhibitor and the training excitor produced an expectancy for US omission that was consistent with the time at which the transfer excitor signaled the forthcoming US. Thus, the present observation of transfer of inhibition by a serial inhibitor is not novel. However in Barnet and Miller's article, an alternative to the temporal coding hypothesis explanation of their results was raised by an anonymous reviewer which, while possible, seemed unlikely. In their study, two groups of animals were trained. In both groups, a serial inhibitor and a simultaneous inhibitor were established, but with different training excitors. For one group, both inhibitors were trained using a delay excitor, and for the other group both inhibitors were trained using an excitor that had been simultaneously paired with the US. At test, the animals trained with the delay training excitor demonstrated inhibition only to the simultaneous inhibitor that had been presented simultaneously with its training excitor (testing was always with a delay transfer excitor). Correspondingly, animals trained with the simultaneous training excitor demonstrated inhibition only to the inhibitor that had been presented serially before its training excitor. No transfer of inhibition was observed with this serially trained inhibitor when the training excitor had been conditioned with a delay relationship with respect to the US, or with the simultaneously trained inhibitor when the training excitor had 
been conditioned with a simultaneous relationship with respect to the US. That is, summation test behavior indicative of inhibition was greatest when the composite temporal relationship of the inhibitor to its training excitor and hence its (omitted) US corresponded to the temporal relationship of the transfer test CS to its US. The alternative explanation suggested to Barnet and Miller by a reviewer was that "the strength of the inhibitory control increases if the inhibitor precedes the otherwise expected US (during training) by up to a few seconds but decreases if the delay gets too long" (Barnet \& Miller, 1996, p. 88). The present results discount this possibility because maximal inhibition was demonstrated with a simultaneous inhibitor established with a training excitor that had been conditioned in a trace relationship to the US (Experiment 1, CY test), and a serial inhibitor established with a training excitor that had been conditioned in a delay relationship to the US. The inhibitor in these two cases was most effective not as a function of when it occurred during testing relative to the occurrence of the expected US (on the basis of the transfer excitors), but as a function of when it predicted the US would be omitted relative to the occurrence of the expected US.

Previous investigations have established that Pavlovian CSs and instrumental discriminative stimuli encode qualitative aspects of the reinforcer (e.g., Colwill \& Motzkin, 1994; Colwill \& Rescorla, 1988). Additionally, instrumental studies of timing suggest that subjects learn when a reinforcer will be presented (Roberts, 1981). The present series of experiments in conjunction with the findings of Barnet and Miller (1996) extend our understanding of associative learning by demonstrating that subjects can also encode when a US will be omitted. The temporal coding hypothesis appears to provide an explanation for the conditions in which inhibition will transfer to other stimuli. Whether it proves more fruitful to use the temporal coding hypothesis as a complement to traditional associative principles or to completely reject traditional associative principles in favor of a model based purely on timing (e.g., Gallistel, 1990) remains to be determined. But evidence is rapidly accumulating that associative models that do not allow for the encoding of temporal relationships are not viable.

\section{References}

Barnet, R. C., Arnold, H. M., \& Miller, R. R. (1991). Simultaneous conditioning demonstrated in second-order conditioning: Evidence for similar associative structure in forward and simultaneous conditioningLearning and Motivation, 22, 253-268.

Barnet, R. C., Cole, R. P., \& Miller, R. R. (1997). Temporal integration in second-order conditioning and sensory preconditioningAnimal Learning \& Behavior, 25, 221-233.

Barnet, R. C., Grahame, N. J., \& Miller, R. R. (1993). Temporal encoding as a determinant off blockingJournal of Experimental Psychology: Animal Behavior Processes, 19, 327-341.

Barnet, R. C., \& Miller, R. R. (1996). Temporal encoding as a determinant of inhibitory controlLearning and Motivation, 27, 73-91. 
Blaisdell, A. P., Denniston, J. C., \& Miller, R. R. (1998). Temporal encoding as a determinant of overshadowingJournal of Experimental Psychology: Animal Behavior Processes, 24, 72-83.

Blaisdell, A. P., Denniston, J. C., \& Miller, R. R. (1997). Unblocking with qualitative change of USLearning and Motivation, 28, 268-279.

Bouton, M. E., \& Nelson, J. B. (1994). Context-specificity of target versus feature inhibition in a feature-negative discriminationJournal of Experimental Psychology: Animal Behavior Processes, 20, 51-65.

Cole, R. P., Barnet, R. C., \& Miller, R. R. (1995). Temporal encoding in trace conditioningAnimal Learning \& Behavior, 23, 144-153.

Colwill, R. M. (1991). Negative discriminative stimuli provide information about the identity of omitted response-contingent outcomesAnimal Learning \& Behavior, 19, 326-336.

Colwill, R. M., \& Motzkin, D. K. (1994). Encoding of the unconditioned stimulus in Pavlovian conditioningAnimal Learning \& Behavior, 22, 384-394.

Colwill, R. M., \& Rescorla, R. A. (1988). Associations between the discriminative stimulus and the reinforcer in instrumental learningJournal of Experimental Psychology: Animal Behavior Processes, 14, 155-164.

Desmond, J. E., \& Moore, J. W. (1988). Adaptive timing in neural networks: The conditioned responseBiological Cybernetics, 58, 405-415.

Gallistel, C. R. (1990). The organization of learning. Cambridge, MA: MIT Press.

Gibbon, J., \& Balsam, P. (1981). Spreading association in time. In C. M.Locurto, H. S.Terrace, \& J.Gibbon (Eds.), Autoshaping and conditioning theory (pp. 219-253). New York: Academic Press.

Goddard, M. J. (1995). Acquisition of US-no US associations in Pavlovian conditioningLearning and Motivation, 26, 264-277.

Goddard, M. J., \& Jenkins, H. M. (1988). Blocking of a CS-US association by a US-US associationJournal of Experimental Psychology: Animal Behavior Processes, 14, 177-186.

Holland, P. C. (1980). Influence of visual conditioned stimulus characteristics on the form of Pavlovian appetitive conditioned responding in ratsJournal of Experimental Psychology: Animal Behavior Processes, 6, 81-97.

Holland, P. C. (1989). Transfer of negative occasion setting and conditioned inhibition across conditioned and unconditioned stimuliJournal of Experimental Psychology: Animal Behavior Processes, 15, 311-328. 
Holland, P. C. (1991). Transfer of control in ambiguous discriminationsJournal of Experimental Psychology: Animal Behavior Processes, 17, 231-248.

Holland, P. C., \& Lamarre, J. (1984). Transfer of inhibition after serial and simultaneous feature negative discrimination trainingLearning and Motivation, 15, 219-243.

Lamarre, J., \& Holland, P. C. (1987). Transfer of inhibition after serial feature negative discrimination trainingLearning and Motivation, 18, 319-342.

Matzel, L. D., Held, F. P., \& Miller, R. R. (1988). Reexamination of simultaneous and backward conditioning: Implications for contiguity theoryLearning and Motivation, 19, 317-344.

Miller, R. R., \& Barnet, R. C. (1993). The role of time in elementary associations Current Directions in Psychological Science, 2, 106-111.

Miller, R. R., Hallam, S. C., Hong, J. Y., \& Dufore, D. S. (1991). Associative structure of differential inhibition: Implications for models of conditioned inhibitionJournal of Experimental Psychology: Animal Behavior Processes, 17, 141-150.

Pavlov, I. P. (1927). Conditioned reflexes. London: Oxford University Press.

Rescorla, R. A. (1967). Inhibition of delay in Pavlovian fear conditioning Journal of Comparative and Physiological Psychology, 64, 114-120.

Roberts, S. (1981). Isolation of an internal clockJournal of Experimental Psychology: Animal Behavior Processes, 7, 242-268.

Schmajuk, N. A., Lamoureux, J. A., \& Holland, P. C. (1998). Occasion setting: A neural network approachPsychological Review, 105, 3-32. 